IJMMS 29:11 (2002) 681-686

PII. S0161171202011390

http://ijmms.hindawi.com

(c) Hindawi Publishing Corp.

\title{
ON A CLASS OF EVEN-DIMENSIONAL MANIFOLDS STRUCTURED BY AN AFFINE CONNECTION
}

\author{
I. MIHAI, A. OIAGĂ, and R. ROSCA
}

Received 16 January 2001 and in revised form 29 June 2001

\begin{abstract}
We deal with a $2 m$-dimensional Riemannian manifold $(M, g)$ structured by an affine connection and a vector field $\mathscr{T}$, defining a $\mathscr{T}$-parallel connection. It is proved that $\mathscr{T}$ is both a torse forming vector field and an exterior concurrent vector field. Properties of the curvature 2-forms are established. It is shown that $M$ is endowed with a conformal symplectic structure $\Omega$ and $\mathscr{T}$ defines a relative conformal transformation of $\Omega$.
\end{abstract}

2000 Mathematics Subject Classification: 53B05, 53C05, 53D05.

1. Introduction. In [5], a class of odd-dimensional manifolds endowed with a $\mathscr{T}$ parallel connection was investigated.

In the present paper, we consider a $2 m$-dimensional Riemannian manifold $(M, g)$, structured by an affine connection defined by the torsion 2-forms $S^{A}, A \in\{1,2, \ldots$, $2 m\}$. If $\left\{e_{A}\right\}$ and $\left\{\omega^{A}\right\}$ are a vector and a covector basis, respectively, and $\mathscr{T}\left(T^{A}\right)$ a vector field (called the structure vector field of $M$ ), we assume that $\mathcal{T}$ defines a $\mathcal{T}$ parallel connection, in the sense of [9] (see also [2, 4]), that is, the connection forms associated with $\left\{e_{A}\right\}$ and $\left\{\omega^{A}\right\}$ satisfy

$$
\theta_{B}^{A}=\left\langle\mathcal{T}, e_{B} \wedge e_{A}\right\rangle=T^{B} \omega^{A}-T^{A} \omega^{B},
$$

where $\wedge$ means the wedge product of vector fields, which implies $\nabla_{g} e_{A}=0$.

Next, we assume that the torsion forms $S^{A}$ are exterior recurrent (abbreviated ER) [1] with $\alpha=\mathscr{T}^{b}$ as recurrence form, that is, $d S^{A}=\alpha \wedge S^{A}$.

Assuming that $T^{A}$ are also ER with a certain Pfaffian $u$ as recurrence form, that is, $d T^{A}=T^{A} u$, and denoting $2 t=\|\mathscr{T}\|^{2}$, we have

$$
\nabla \mathscr{T}=2 t d p+(u-\alpha) \otimes \mathscr{T}
$$

where $d p$ is the soldering form of $M$ [3], which says that $\mathscr{T}$ is a torse forming vector field $[8,11,12]$.

We derive

$$
\nabla^{2} \mathscr{T}=2 t(u+\alpha) \wedge d p
$$

that is, $\mathscr{T}$ is an exterior concurrent vector field [10] (see also [4]).

Setting $S=S^{1} \wedge S^{2} \wedge \cdots \wedge S^{2 m}$, we find that the $4 m$-form $S$ associated with $M$ is ER with $4 m \alpha$ as recurrence form. 
It is shown that the curvature 2-forms $\Theta_{B}^{A}$ are ER having the closed 1-form 2( $u+$ $\alpha)$ as recurrence form. We agree to define such a manifold as an exterior recurrent curvature 2-form manifold.

Finally, assuming that $M$ carries an almost symplectic form $\Omega$, that is, a nondegenerate differential 2-form, we prove that $\Omega$ is a conformal symplectic form.

It is shown that $\mathcal{T}$ defines a relative conformal transformation of the conformal symplectic form $\Omega$ (see [5]).

The above results are stated in Theorem 3.1.

2. Preliminaries. Let $(M, g)$ be a $2 m$-dimensional oriented Riemannian manifold structured by an affine differential operator $\nabla$.

Let $\Gamma(T M)$ be the set of sections of the tangent bundle and $b: T M \rightarrow T^{*} M$ and $\#: T^{*} M \rightarrow T M$ the classical musical isomorphisms defined by $g$ (i.e., $b$ is the index lowering operator and $\#$ is the index raising operator).

Following [7], we denote by

$$
A^{q}(M, T M)=\Gamma \operatorname{Hom}\left(\wedge^{q} T M, T M\right)
$$

the set of vector-valued $q$-forms $(q \leq \operatorname{dim} M)$ and we write for the affine operator $\nabla$

$$
d^{\nabla}: A^{q}(M, T M) \longrightarrow A^{q+1}(M, T M) .
$$

If $d p \in A^{1}(M, T M)$ is the canonical vector-valued 1 -form of $M$, then as an extension of the Levi-Civita operator and by [3], we agree to call $d p$ the soldering form of $M$.

Let the unit vector fields $\left\{e_{A}\right\}$ be an orthonormal vector basis and $\left\{\omega^{A}\right\}$ its corresponding cobasis on $M, A=1, \ldots, 2 m$. Then, if $\theta_{B}^{A}, S^{A}$, and $\Theta_{B}^{A}$ denote the connection forms, the torsion 2-forms and the curvature 2-forms, respectively, Cartan's structure equations are expressed by

$$
\begin{aligned}
\nabla e_{A} & =\theta_{B}^{A} \otimes e_{B}, \\
d \omega^{A} & =\omega^{B} \wedge \theta_{B}^{A}+S^{A}, \\
d \theta_{B}^{A} & =\theta_{B}^{C} \wedge \theta_{C}^{A}+\Theta_{B}^{A} .
\end{aligned}
$$

We recall the following definitions (cf. [4]).

A vector field $\mathcal{T}$ is said to be a torse forming vector field [12] if it satisfies

$$
\nabla \mathscr{T}=f \mathscr{T}+v \otimes \mathscr{T}, \quad f \in C^{\infty} M, v \in \wedge^{1} M .
$$

Also, the vector field $\mathscr{T}$ is called exterior concurrent [10] if

$$
\nabla^{2} \mathscr{T}=\pi \wedge d p, \quad \pi \in \wedge^{1} M .
$$

If $Z, Z^{\prime} \in \Gamma(T M)$, we also have the following formula:

$$
d \omega\left(Z, Z^{\prime}\right)=\mathscr{L}_{Z^{\prime}} \omega(Z)-\mathscr{L}_{Z} \omega\left(Z^{\prime}\right)+\omega\left(\left[Z, Z^{\prime}\right]\right),
$$

where $\mathscr{L}$ is the Lie derivative. 
Since $d p=\omega^{A} \wedge e_{A}$, then it follows that

$$
d^{\nabla}(d p)=S^{A} \otimes e_{A}
$$

3. Manifolds with affine connection. In the present paper, we assume first that the $2 m$-dimensional Riemannian manifold $(M, g)$ carries a structure vector field $\mathscr{T}\left(T^{A}\right)$ which defines a $\mathcal{T}$-parallel connection, in the sense of [9] (see also [2, 4]). Such a connection is expressed by

$$
\theta_{B}^{A}=\left\langle\mathscr{T}, e_{B} \wedge e_{A}\right\rangle=T^{B} \omega^{A}-T^{A} \omega^{B} .
$$

Since we quickly find from (3.1) that

$$
\nabla_{\mathscr{T}} e_{A}=0
$$

this agrees with the definition of $\mathscr{T}$-parallel connection.

Setting $2 t=\|T\|^{2}$, we derive

$$
\nabla \mathscr{T}=2 t d p-\alpha \otimes \mathscr{T}+\sum_{A} d T^{A} \otimes e_{A}
$$

where $\alpha=\mathscr{T}^{b}$ is the dual 1 -form of $\mathscr{T}$. Also, we find by (3.1) and (2.4) that

$$
d \omega^{A}=\alpha \wedge \omega^{A}+S^{A} .
$$

Second, we assume that the torsion forms $S^{A}$ are exterior recurrent [1] having $\alpha$ as recurrence form, that is,

$$
d S^{A}=\alpha \wedge S^{A}
$$

and $T^{A}$ are ER with the Pfaffian $u$ as recurrence form, that is,

$$
d T^{A}=T^{A} u
$$

We obtain $d \alpha=0$, that is, $\alpha^{\sharp}=\mathscr{T}$ is a closed vector field.

Under these conditions, it follows from (3.3) and (3.6) that

$$
\nabla \mathscr{T}=2 t d p+(u-\alpha) \otimes \mathscr{T}
$$

this proves that $\mathscr{T}$ is a torse forming vector field $[4,8,11,12]$. Since the operator $\nabla$ acts inductively and clearly by (3.6), then

$$
d t=2 t u
$$

we infer

$$
d^{\nabla}(\nabla \mathscr{T})=\nabla^{2} \mathscr{T}=2 t(u+\alpha) \wedge d p
$$

This means that the vector field $\mathcal{T}$ is an exterior concurrent vector field $[6,10]$. 
By [6], (3.9) implies that

$$
\mathscr{R}(\mathscr{T}, Z)=-(2 m-1) 2 \operatorname{tg}(\mathscr{T}, Z), \quad Z \in \Gamma(T M),
$$

where $\mathscr{R}$ denotes the Ricci tensor field on $M$.

By (3.9) and by standard calculation, we derive

$$
\nabla^{4} \mathscr{T}=0
$$

and therefore we may say that the vector field $\mathscr{T}$ is an element of

$$
\Gamma \operatorname{Hom}\left(\wedge^{4} T M, T M\right) .
$$

On the other hand, recall that the Bianchi forms in the sense of Tachibana are defined by

$$
\Omega_{\alpha_{1}, \ldots, \alpha_{2 p}}^{(p)}=\Omega_{\alpha_{1}}^{\alpha_{2}} \wedge \Omega_{\alpha_{2}}^{\alpha_{3}} \wedge \cdots \wedge \Omega_{\alpha_{2 p-1}}^{\alpha_{2 p}}
$$

where $\Omega_{\alpha_{q}}^{\alpha_{q+1}}$ are 2-forms. Thus, setting

$$
S=S^{1} \wedge S^{2} \wedge \cdots \wedge S^{2 m}
$$

we find that

$$
d S=4 m \alpha \wedge S
$$

Therefore, we may say that the $4 m$-form $S$ associated with $M$ is ER with $4 m \alpha$ as recurrence form.

By (3.4) we may set

$$
S^{A}=u \wedge \omega^{A}
$$

and by (3.1) and the structure equations (2.5) we get after some calculations

$$
\Theta_{B}^{A}=2(u+\alpha) \wedge \omega_{B}^{A}+2 t \omega^{B} \wedge \omega^{A} .
$$

Next, performing the exterior differentiation of $\Theta_{B}^{A}$, we derive, taking account of (3.8)

$$
d \Theta_{B}^{A}=2(u+\alpha) \wedge \Theta_{B}^{A} .
$$

This shows that all curvature forms $\Theta_{B}^{A}$ are ER and have the closed 1-form $2(u+\alpha)$ as recurrence form.

We agree to define such an even-dimensional manifold $M$ as an exterior recurrent curvature 2-form manifold.

Finally, assume that $M$ carries an almost symplectic form $\Omega$. Then, we may express $\Omega$ as

$$
\Omega=\sum_{a=1}^{m} \omega^{a} \wedge \omega^{a^{*}}, \quad a^{*}=a+m .
$$


Taking the exterior differentiation of $\Omega$, we find by (3.4) and (3.16) that

$$
d \Omega=2(\alpha+u) \wedge \Omega .
$$

This shows that the manifold under consideration is endowed with a conformal symplectic structure having $\alpha+u$ as covector of Lee.

Moreover, taking the Lie differentiation of $\Omega$ with respect to the structure vector field $\mathscr{T}$, we infer

$$
\mathscr{L}_{\mathscr{J}} \Omega=u t \Omega+2(u+\alpha) \wedge \sum_{a=1}^{m}\left(T^{a} \omega^{a^{*}}-T^{a^{*}} \omega^{a}\right) .
$$

Using (3.8) and (3.6), the exterior differentiation of (3.21) gives

$$
d \mathscr{L}_{\mathscr{T}} \Omega=8 t u \wedge \Omega
$$

Hence, by [4], the above equation says that $\mathscr{T}$ defines a relative conformal transformation of the conformal symplectic form $\Omega$.

Summing up, we state the following theorem.

THEOREM 3.1. Let $(M, g)$ be a 2 m-dimensional Riemannian manifold structured by an affine connection defined by the torsion 2-forms $S^{A}, A=1, \ldots, 2 m$. Let $\mathcal{T}\left(T^{A}\right)$ be a structure vector field, which defines a $\mathcal{T}$-parallel connection and assume that $S^{A}$ are exterior recurrent, having $\mathscr{T}^{b}$ as recurrence form $\left(\mathcal{T}^{b}=\alpha\right.$ is a closed Pfaffian).

Then the following properties hold:

(i) $\mathcal{T}$ is both a torse forming and an exterior concurrent vector field;

(ii) the structure curvature 2-forms $\Theta_{B}^{A}$ are exterior recurrent with the closed Pfaffian $2(u+\alpha)$ as recurrence form;

(iii) the manifold $M$ is endowed with a conformal symplectic structure $\Omega$ having $u+\alpha$ as covector of Lee;

(iv) the vector field $\mathcal{T}$ defines a relative conformal transformation of $\Omega$, that is, $d \mathscr{L}_{\mathscr{T}} \Omega=8$ tu $\wedge \Omega$, where $2 t=\|\mathscr{T}\|^{2}$.

\section{REFERENCES}

[1] D. K. Datta, Exterior recurrent forms on a manifold, Tensor (N.S.) 36 (1982), no. 1, 115120.

[2] F. Defever and R. Rosca, On a class of even-dimensional manifolds structured by a $\mathcal{T}$ parallel connection, Tsukuba J. Math. 25 (2001), no. 2, 359-369.

[3] J. Dieudonné, Treatise on Analysis. Vol. IV, Pure and Applied Mathematics, vol. 10-IV, Academic Press, New York, 1974.

[4] I. Mihai, R. Rosca, and L. Verstraelen, Some Aspects of the Differential Geometry of Vector Fields. On Skew Symmetric Killing and Conformal Vector Fields, and Their Relations to Various Geometrical Structures, Centre for Pure and Applied Differential Geometry (PADGE), vol. 2, Katholieke Universiteit Brussel Group of Exact Sciences, Brussels, 1996.

[5] I. Mihai, L. Verstraelen, and R. Rosca, On a class of exact locally conformal cosymplectic manifolds, Int. J. Math. Math. Sci. 19 (1996), no. 2, 267-278.

[6] M. Petrović, R. Rosca, and L. Verstraelen, Exterior concurrent vector fields on Riemannian manifolds. I. Some general results, Soochow J. Math. 15 (1989), no. 2, 179-187. 
[7] W. A. Poor, Differential Geometric Structures, McGraw-Hill, New York, 1981.

[8] R. Rosca, On exterior quasi concurrent and on torse forming vector fields on a Riemannian manifold, in preparation.

[9]___ On parallel conformal connections, Kodai Math. J. 2 (1979), no. 1, 1-10.

[10] _ Exterior concurrent vector fields on a conformal cosymplectic manifold endowed with a Sasakian structure, Libertas Math. 6 (1986), 167-174.

[11] C. Udrişte, Properties of torse-forming vector fields, Tensor (N.S.) 42 (1985), no. 2, 137 144.

[12] K. Yano, On the torse-forming directions in Riemannian spaces, Proc. Imp. Acad. Tokyo 20 (1944), 340-345.

I. Mihai: Faculty of MATHematics, Str. ACADEmiei 14, 70109 Bucharest, RomaniA

E-mail address: imi hai@math . math . unibuc . ro

A. Oiagă: Faculty of Mathematics, STR. ACAdemiei 14, 70109 Bucharest, Romania

E-mail address: ade1a@geometry . math . unibuc. ro

R. RosCA: 59 Avenue Emile Zola, 75015 PARIS, FrancE 


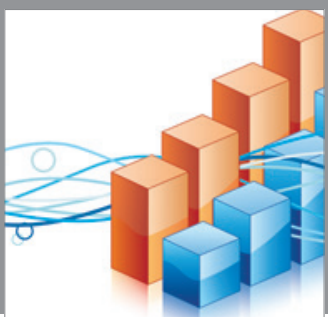

Advances in

Operations Research

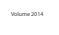

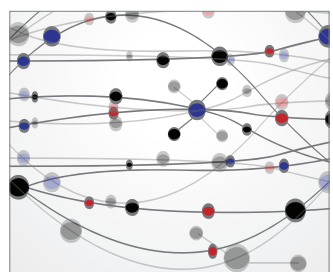

\section{The Scientific} World Journal
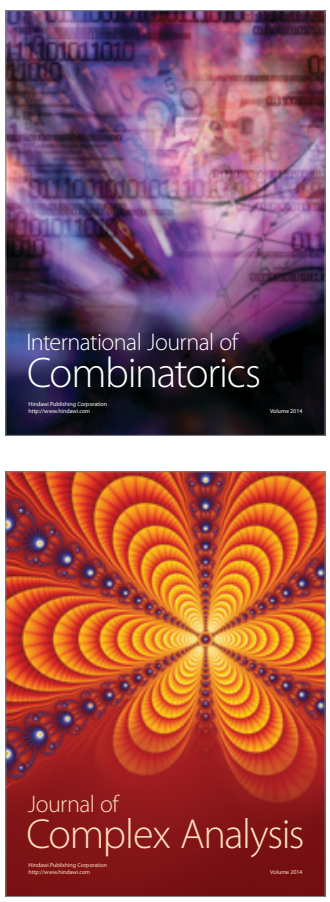

International Journal of

Mathematics and

Mathematical

Sciences
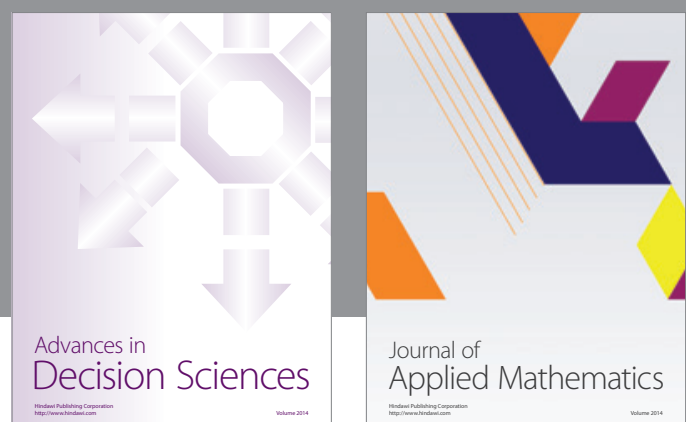

Journal of

Applied Mathematics
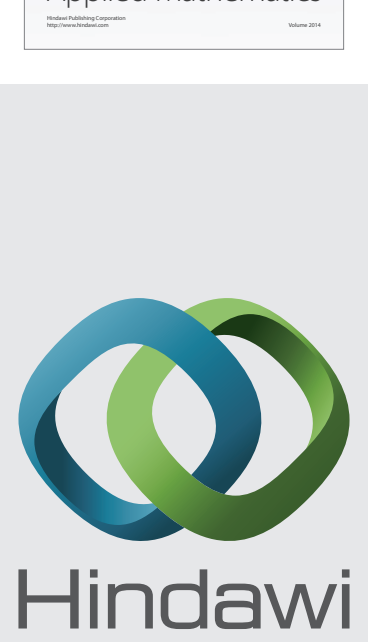

Submit your manuscripts at http://www.hindawi.com
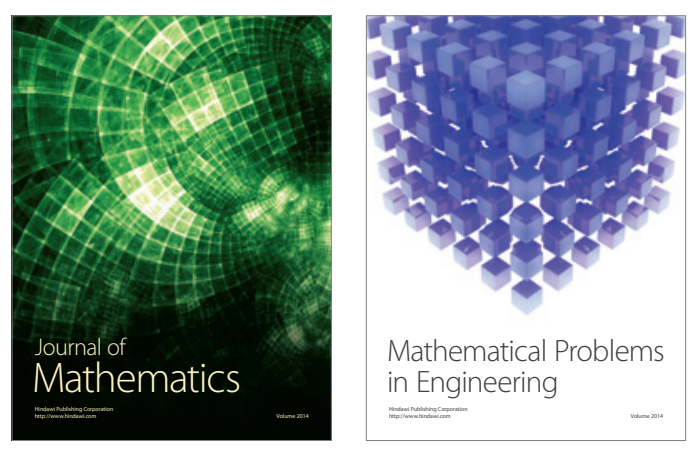

Mathematical Problems in Engineering
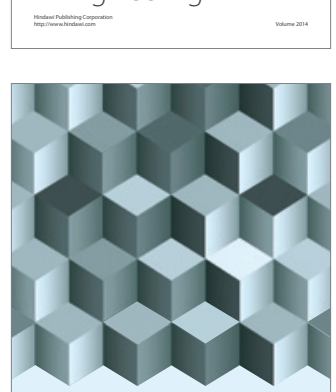

Journal of

Function Spaces
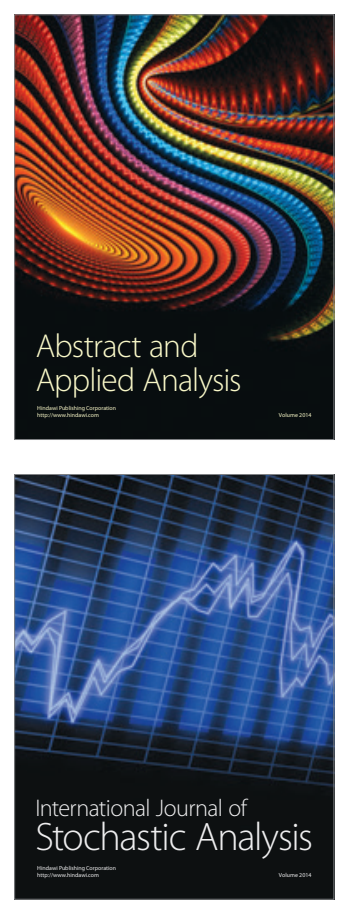

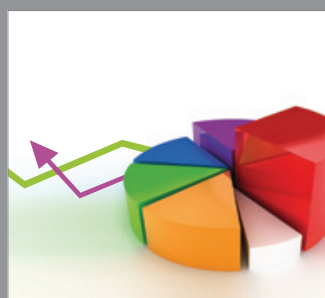

ournal of

Probability and Statistics

Promensencen
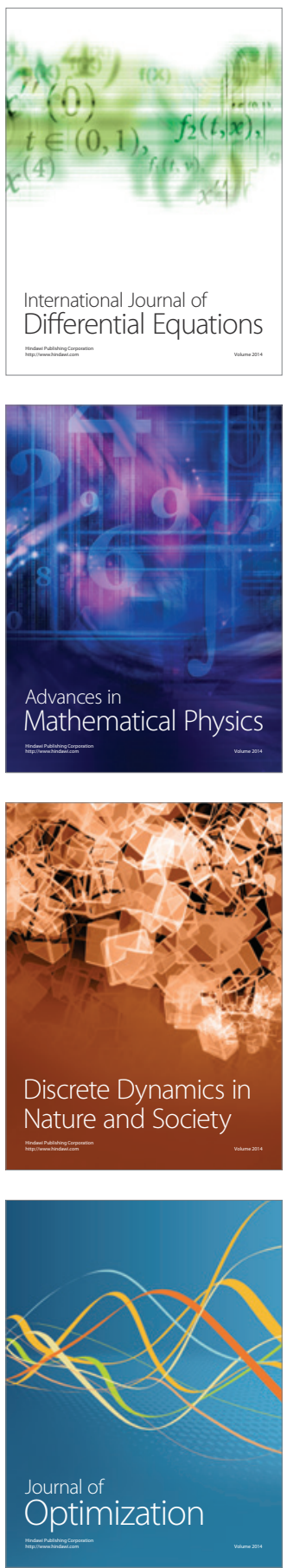